\title{
THE EFFECT OF VISIBLE LIGHT IRRADIATION ON POSITRONIUM FORMATION IN POLYETHYLENE AT LOW TEMPERATURE
}

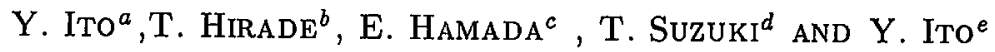 \\ ${ }^{a}$ High Energy Accelerator Research Organization (KEK) \\ Tanasi-Branch, 3-2-1 Midori, Tanasi-si, Tokyo 188-8501, Japan \\ ${ }^{b}$ Department of Materials Science, Japan Atomic Energy Research Institute \\ Tokai, Naka, Ibaraki 319-1106, Japan \\ ${ }^{c}$ The Graduate University for Advanced Studies \\ High Energy Accelerator Research Organization (KEK) \\ 1-1, Oho, Tsukuba, Ibaraki 305-0801, Japan \\ ${ }^{d}$ High Energy Accelerator Research Organization (KEK) \\ 1-1, Oho, Tsukuba, Ibaraki 305-0801, Japan \\ ${ }^{e}$ Research Center for Nuclear Science and Technology, University of Tokyo \\ Tokai, Naka, Ibaraki 319-1106, Japan
}

The effect of visible light exposing on the ortho-positronium formation in polyethylene was studied. Lifetime spectra of positrons in polyethylene were measured in the temperature range between $50 \mathrm{~K}$ and $370 \mathrm{~K}$. It was observed that the visible light quenches the enhancement of ortho-positronium intensity $\left(I_{3}\right)$ at lower temperature than ca. $250 \mathrm{~K}$ - the glass transition temperature of polyethylene. On the other hand, the lifetime of ortho-positronium component $\left(\tau_{3}\right)$ was not affected by the presence of the visible light.

PACS numbers: $78.70 . \mathrm{Bj}$

\section{Introduction}

It is well known that, in the most of polymers, lifetime spectra of annihilating positrons have three components. The longest lifetime component $\left(\tau_{3}\right)$ is assigned to the pick-off annihilation of ortho-positronium (o-Ps) localized in intermolecular spaces (holes) and there is a simple relation between $\tau_{3}$ and the size of the holes $[1,2]$. Although it has been recognized that $o$-Ps intensity $\left(I_{3}\right)$ is related to the amount of the intermolecular spaces, the temperature dependence of $I_{3}$ is not always explained by a simple relation. Many publications have been devoted to the study of polymers behavior at low temperature [3-8].

From the glass transition temperature $\left(T_{\mathrm{g}}\right)$ to room temperature, $I_{3}$ is known to increase with increasing temperature. It is one of the explanations for these phenomena that the size of intermolecular spaces and their concentration are increased. with temperature by the micro-Brownian motion of molecules. On the other hand, 
it has been reported that, below $T_{\mathrm{g}}$, the $I_{3}$ increased along with decreasing temperature. Such a dependence has been considered as caused by the freezing of the local motion of molecules and the several models are proposed as follows $[3,4]$ : because of the local motion, the electron density distribution in the lattice is smeared out, resulting in the high electron local density in the free volume, and this higher local electric field can reduce the o-Ps yield; the preexisting holes are shielded by local motions and o-Ps can migrate into the hole only at low temperature after the freezing of the motions.



Fig. 1. Positron irradiation effect on polypropylene kept at several low temperatures. The sample was cooled to each of them within 50 minutes.

In addition, it is known that another correlation exists at low temperature. As discussed in Refs. [3,7-9], $I_{3}$ slowly increases together with the time after cooling and it takes a few days to reach the equilibrium. The typical increase in $I_{3}$ for polypropylene under different cooling temperatures was reported as shown in Fig. 1 [9]. It reveals that the increasing rate depends on temperature, $I_{3}$ increases faster at lower temperature and $I_{3}$ does not increase at $240 \mathrm{~K}$ in polypropylene. This phenomenon had been explained by the relaxation of internal polymer structures at low temperature [10]. In addition to such observation, recently, an interesting decrease in $I_{3}$ in poly-methyl-methacrylate (PMMA) at low temperature was observed with exposing the visible light by Hirade et al. [11]. This effect suggests that it is inadequate to associate $I_{3}$ simply with the amount of intermolecular spaces and the relaxation of polymer structures at low temperature. We report here that such a visible light effect is also seen for polyethylene (PE) at low temperature. 


\section{Experimental}

The effect of visible light irradiation on the o-Ps formation was studied by the positron annihilation lifetime spectroscopy (PALS). A conventional fast-fast coincidence system was used for measuring the time spectra. The time resolution of the system was $c a .280$ ps (the full width at half maximum). Decomposition of lifetime spectra into three exponential components was performed using PATFIT [12].

The PE samples used in this study were commercially available as high density PEs produced by Mitsui Petrochemical Industries and kindly supplied by that company. The characteristics are as follows: density $\sim 0.968 \mathrm{~g} / \mathrm{cm}^{3}$; melting point $\sim 407 \mathrm{~K}$; crystallinity $\sim 0.66$; molecular weight $\sim 6 \times 10^{4}$; ratio of branches of $-\mathrm{CH}_{3}$ per 100 carbon atoms $\sim 1.0$. These samples were used without any pretreatment like annealing above $T_{\mathbf{g}}$ before this experiment. A positron source of about $1 \mathrm{MBq}(\mathrm{ca} .30 \mu \mathrm{Ci}){ }^{22} \mathrm{Na}$ was sandwiched between two PE samples with the size of $12 \times 15 \times 1 \mathrm{~mm}^{3}$, and placed inside a refrigerator. Several thermocouples (TC) placed inside the refrigerator being served to measure and control temperatures. An $\mathrm{Au}-\mathrm{Cr}(\mathrm{Fe}) \mathrm{TC}$ was placed $5 \mathrm{~mm}$ below the source in order to measure the temperature between the two samples.

The samples were exposed to the visible light guided from outside of the refrigerator by an optical fiber with a diameter of $1 \mathrm{~mm}$. The fiber was placed between two samples and located $10 \mathrm{~mm}$ above the source. The visible light was produced by a xenon lamp and then focused by lens to enhance the density of light.

\section{Results and discussion}

The $I_{3}$ and $\tau_{3}$ in PE are shown in Fig. 2 as a function of elapsed time. Open and solid symbols represent $I_{3}$ and $\tau_{3}$, respectively. The solid line represents the temperature control of the refrigerator. At the beginning, the temperature was kept at $300 \mathrm{~K}$ for three hours, then, PE was cooled gradually within 50 minutes from $300 \mathrm{~K}$ to $80 \mathrm{~K}$ in the dark. From $5 \mathrm{~h}$ to $76 \mathrm{~h}$, the temperature of the sample was kept at $80 \mathrm{~K}$. At $72 \mathrm{~h}$, the sample was exposed by the visible light, then, the temperature was increased slowly, $8 \sim 9 \mathrm{~K} / \mathrm{h}$, from $80 \mathrm{~K}$ to $370 \mathrm{~K}$ in the presence of visible light.

As shown in Fig. 2, between $5 \mathrm{~h}$ and $71 \mathrm{~h}$, the increase in $I_{3}$ along with the elapsed time was observed. As soon as PE was exposed to the visible light at $72 \mathrm{~h}$, the sharp decrease in $I_{3}$ occurred. This phenomenon suggests that the o-Ps formation is strongly obstructed by the presence of the visible light.

The temperature dependence of $I_{3}$ and $\tau_{3}$ is shown in Fig. 3. Open and solid symbols represent data with and without exposing the samples to the visible light, respectively. Below $250 \mathrm{~K}$, the decrease in $I_{3}$ with the visible light exposing was clearly observed. Commonly, $T_{\mathrm{g}}$ of PE is distributed between $220 \mathrm{~K}$ and $270 \mathrm{~K}$ [10]; and from Fig. $3 T_{\mathrm{g}}$ for PE used in this experiment is estimated to be around $250 \mathrm{~K}$. From $250 \mathrm{~K}$ to $370 \mathrm{~K}$, the effect of visible light exposure was not seen. On the other hand, $\tau_{3}$ was not affected by the presence of the visible light at all. These results suggest that the effect of visible light is seen only on Ps formation at lower temperature than $T_{\mathrm{g}}$ for $\mathrm{PE}$. 


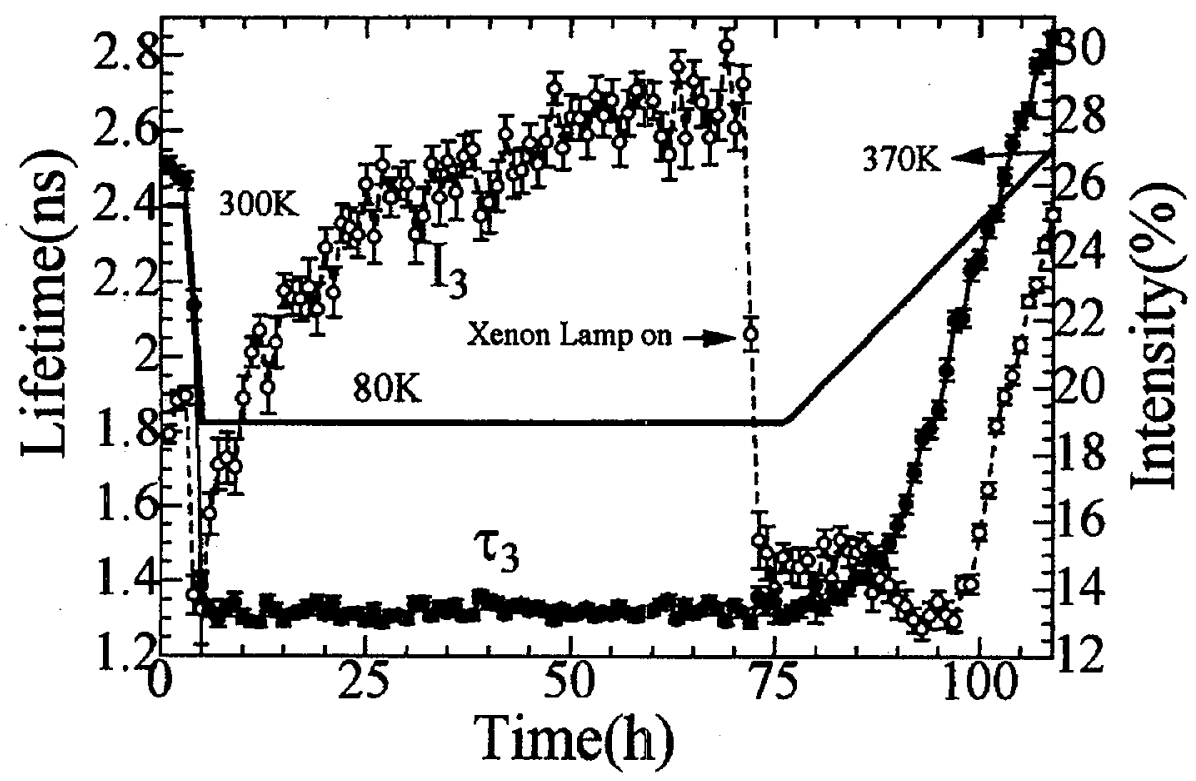

Fig. 2. Positron irradiation effect on polyethylene versus the elapsed time. The temperature control was done as follows: from 0 to $3 \mathrm{~h}, 300 \mathrm{~K}$; from $4 \mathrm{~h}$ to $76 \mathrm{~h}, 80 \mathrm{~K}$; heating from $77 \mathrm{~h}$ to $109 \mathrm{~h}$ at $8 \sim 9 \mathrm{~K} / \mathrm{h}$. The sample was exposed to visible light from $72 \mathrm{~h}$ to $109 \mathrm{~h}$. Open and solid symbols show the intensity and the lifetime of o-Ps, respectively.

In addition to PMMA [11], we have observed the visible light effect on the Ps formation in PE. Hirade et al. [11] explained this effect as follows. It is known by electron paramagnetic resonance study [13] that the weakly bound electron is stable below $170 \mathrm{~K}$, probably the transition temperature, where the local molecular motions are frozen, and the enhancement of $I_{3}$ is also observed below the temperature [3-8]. Moreover, the visible light, the energy of $2 \sim 3 \mathrm{eV}$, can bleach the weakly bound electrons even below $170 \mathrm{~K}$ [13]. The bounding energy of the weakly bound electrons should be less than $2 \sim 3 \mathrm{eV}$. These electrons can be picked off by positrons to form Ps, because the bounding energy of Ps is $6.8 \mathrm{eV}$ in vacuum and, probably, $3 \sim 4 \mathrm{eV}$ in polymers.

For PE, above $T_{\mathrm{g}}$, the enhancement was not observed because the trapped electrons are easily detrapped by the three-dimensional molecular motion of main chain of polymer.

The electron may be trapped at the shallow trap by polarization forces [14] which is, probably, not anion type like in PMMA. The absorption band of the trapped electrons in $\mathrm{PE}$ extends from the edge of the vacuum ultraviolet rays to well into the infrared, and the trapping sites are mainly in the crystalline phase [14].

We observed the same effect by exposing $\mathrm{PE}$ to a red laser light $(633 \mathrm{~nm}$ $\mathrm{He}-\mathrm{Ne}$ laser). Because this light is also in the absorption band of $\mathrm{PE}$ as mentioned above, the weakly bound electrons can be bleached even by a light with an energy of $c a .2 \mathrm{eV}$. 


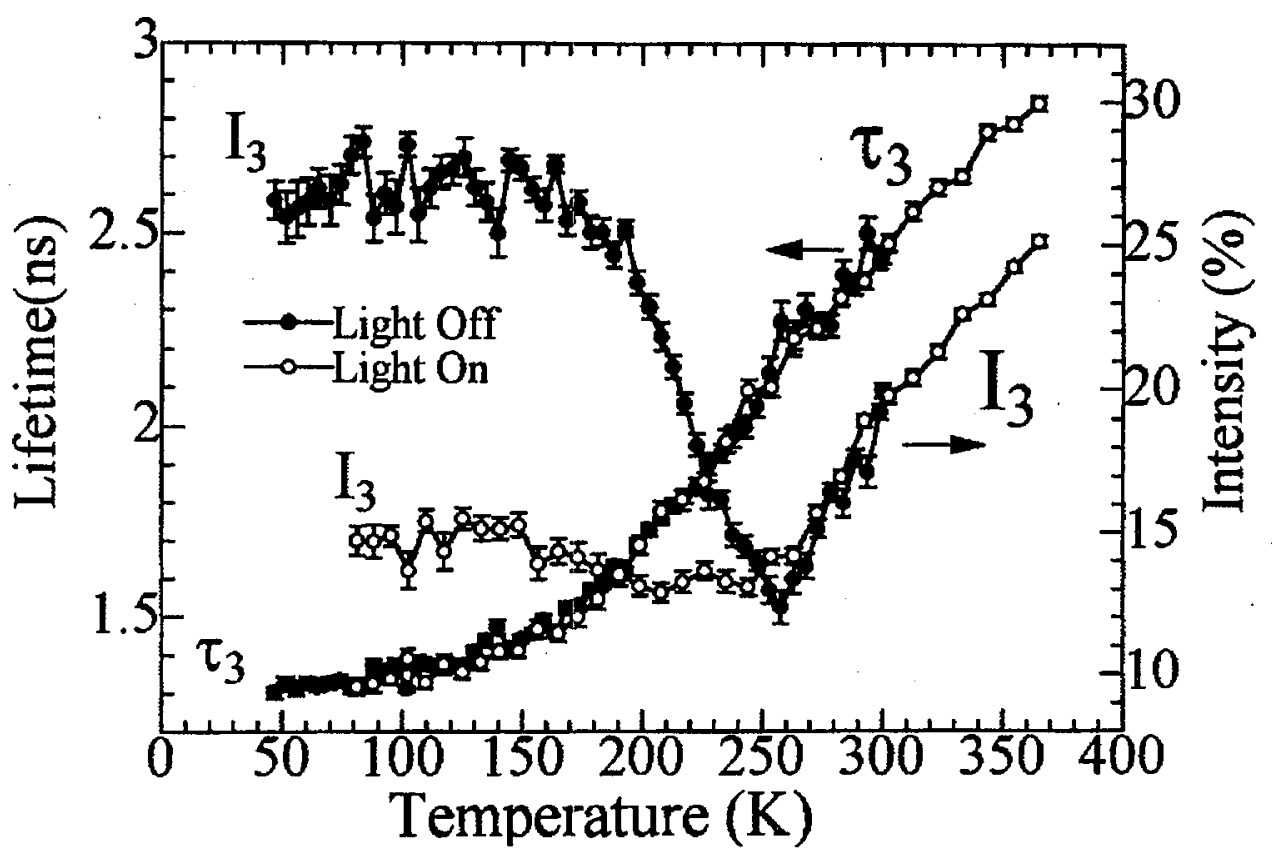

Fig. 3. Positron irradiation effect on polyethylene versus temperature with (open symbol) and without (solid symbol) exposing to the visible light.

\section{Conclusion}

The visible light effect on Ps formation in PMMA was reported by Hirade et al. [11] and we also observed the same effect in PE. Below the glass transition temperature, the increase in $I_{\mathbf{3}}$ along with decreasing temperature is interpreted by the increase in concentration of the trapped electrons, where the electrons are induced in samples by the positron irradiation during the PALS measurement. The trapped electrons in $\mathrm{PE}$ can be bleached by the visible light exposing, resulting in the sharp decrease in $I_{3}$. Since the effect was also observed with the $\mathrm{He}-\mathrm{Ne}$ laser light, these electrons can be removed by a light with an energy of $c a .2 \mathrm{eV}$. The results of the visible light exposure on PE were successfully explained by the model proposed by Hirade et al. [11] based on the spur model instead of the other models proposed before.

\section{Acknowledgment}

This work was supported by the Grant-in-Aid in Scientific Research by the Japanese Ministry of Education, Culture, Sports and Science.

\section{References}

[1] M. Eldrup, D. Lightbody, N. Sherwood, Chem. Phys. 63, 51 (1981).

[2] H. Nakanishi, Y.C. Jean, Positron and Positronium Chemistry, Eds. M. Schrader, Y.C. Jean, Elsevier, New York 1988, p. 159. 
[3] P. Kindl, G. Reiter, Phys. Status Solidi A 104, 707 (1987).

[4] B. Lévay, M. Lalović, H.J. Ache, J. Chem. Phys. 90, 3282 (1989).

[5] A. Uedono, T. Kawano, S. Tanigawa, M. Ban, M. Kyoto, T. Uozumi, J. Polym. Sci. B 34, 2145 (1996).

[6] T. Ogawa, T. Suzuki, M. Murakami, J. Polym. Sci. B 36, 755 (1998).

[7] C.L. Wang, T. Hirade, F.H.J. Maurer, J. Chem. Phys. 108, 4654 (1998).

[8] T. Suzuki, Y. Oki, M. Numajiri, T. Miura, T. Kondo, N. Oshima, Y. Ito, Polymer 37, 5521 (1996).

[9] T. Suzuki, Y. Oki, M. Numajiri, T. Miura, T. Kondo, Y. Ito, J. Phys. IV (France) 3, 283 (1993).

[10] G.T. Davis, R.K. Eby, J. Appl. Phys. 44, 4274 (1973).

[11] T. Hirade, C.L. Wang, F.H. Maurer, M. Eldrup, N.J. Pederson, in: 35th Annual Meeting on Radioisotopes in the Physical Science and Industries, Tokyo (Japan) 1998, Abstracts, p. 89.

[12] P. Kirkegaard, M. Eldrup, O.E. Mogensen, N. Pedersen, Comput. Phys. Commun. 23, 307 (1981) (PATFIT 88, Ris $\varnothing-\mathrm{M}-2740$ (1989 version)).

[13] T. Ichikawa, H. Yoshida, J. Polymer Sci., Part A: Polymer Chemistry 28, 1185 (1990).

[14] R.M. Keyser, K. Tsuji, F. Williams, The Radiation Chemistry of Macromolecules, Ed. M. Dole, Vol. 1, Academic Press, New York 1972, Ch. 9. 